\title{
Towards Integrating Aesthetic Variables in Architectural Design Optimization
}

\author{
SHERMEEN YOUSIF \\ Texas A\&M University
}

\author{
MARK CLAYTON \\ Texas A\&M University
}

\author{
WEI YAN \\ Texas A\&M University
}

This work suggests an approach to assure generation of aesthetically pleasing design candidates with high technical performance. By employing an aesthetically-driven initial population as input to an evolutionary algorithm using technically-oriented fitness functions and a filter to assure diversity, the method guides the algorithm toward candidate solutions that are both aesthetically pleasing and technically proficient. The aim is to overcome criticism of design automation systems that accomplish high technical performance but low aesthetic quality. The method involves review of related research, prototyping a software, and testing the prototype with one or more tests. The prototype, under development, first utilizes parametric modeling for form generation. Aesthetic parameters are introduced to generate the initial population in two steps: (1) creating analytical diagrams that expresses aesthetic ideas derived from architectural theory, and (2) generating synthetic models through parameterization of the aesthetic variables. As a test-case, the process of analytical diagrams devises aesthetic parameters from the modern language of architecture of Bruno Zevi, applying them to a test-case of Richard Meier's Atheneum. Next, a Multi-Objective Evolutionary Algorithm that employs simulation-based fitness functions to optimize the environmental performance will be incorporated into the workflow. A form diversity algorithm that has been developed in a prior work will be incorporated to ensure that redundant forms are eliminated from the candidate set and condense the candidate list into distinctly different candidates. The workflow can then loop back to another generation in the evolutionary algorithm or terminate with a set of Pareto optimum solutions with high aesthetic performance. This research plan is under implementation.

\section{INTRODUCTION}

Architectural design is a complex process that requires the use of different tools and methods to produce and assess form in response to building functional requirements such as program, structure, and environmental performance, in addition to essential design aspects of aesthetics, and sociological and psychological impacts. ${ }^{10}$ In particular, aesthetics in building design yield direct impact on quality of life. ${ }^{6,20}$ The aim of building morphology is mainly the generation of form which is a crucial step in the early stages of design. ${ }^{35}$ On the other hand, architectural choices on the physical building forms and their systems must be made to simultaneously satisfy potentially conflicting objectives and can be treated as a Multi-Objective Optimization (MOO) process. ${ }^{27}$ In such an optimization, rating design options according to only performance often does not produce interesting forms; ${ }^{7}$ additional spatial prescriptions of the quality (aesthetics) of solution candidates are needed. ${ }^{35}$ Form aesthetics should be integrated into optimization as a primary design objective. ${ }^{36}$ Architectural design methods can equally benefit from the combination of optimization methods and quality assessment of generated forms. ${ }^{35}$ Current research experiments confirm the capabilities of using comuptational tools to significantly expedite building design to follow a particular architectural style yet in a performance-driven approach to achieve both aesthetic goals and efficiency. ${ }^{14}$

Identified in related literature are two problems. first, a successful MOO process for building design should meet the aesthetic expectations of creative and expressive forms. ${ }^{8} \mathrm{~A}$ major issue in current architectural optimization models is a lack of aesthetic consideration where the focus is more on the quantifiable objectives. Second, due to slight changes in their parameters, building forms that result from a generative system can be similar and redundant. Checking several similar candidates can be computationally expensive and may overwhelm designers when interacting with the optimization platform. There is still a need for methods that facilitate designers' interaction by decreasing an unwanted number of design solutions that may be generated in automated optimization platforms. ${ }^{38}$

To address the first problem, designers can formalize aesthetic principles into representations and algorithms to be incorporated into the optimization process. In our work, we utilize the aesthetic variables of the formal language of modern architecture to evolve "automatic diagrams". Automatic diagrams are defined as abstracted representations of a scheme that are generated from the Evolutionary Algorithm run, after using aesthetic variables that have been interpreted into constraints/ parameters in form making. Architectural diagrams are equally significant in both functional studies and aesthetic studies. ${ }^{11}$ Aesthetic principles can be diagrammed to effectively highten awareness of aesthetics and provide designers with a tool by which aesthetic ideas can be invented and refined. ${ }^{24}$ Diagrams can be used at the beginning of design process to isolate ideas and improve design cognition, and after design to isolate main concepts in desribing architecture through post-analysis. ${ }^{12}$

To solve the second problem, when generating variant building forms, it is important to guide the evolutionary process to produce highly diverse forms and avoid having 
to analyze similar solutions. One could develop and utilize an algorithm to compare the generated building forms and reduce design options to only distinctly variant solutions. This will help reduce computation time while considering diverse design options. In addition, the reduced set of highly diverse forms may enhance interaction by designers and facilitate decision-making.

We aim to create a parametric and constraint-based prototype that may be capable of expressing key aspects of aesthetics, allowing tests of conformance with formal principles of a style while simultaneously allowing analysis of building performance. Our investigation has implications for computational design process in education and practice.

The paper is organized as follows. The background section gives an overview of the existing work and introduction to the main concepts and technical areas. The methods part introduces the workflow, focusing on prototype development and the test-case. The prototypical protocol consists of three main phases: form generation, optimization, and form comparison. This paper is focused on introducing the prototype, and the form generation phase. Under-progress, is the optimization and application of form diversity algorithm of the test-case. Finally, discussion, conclusions and research significance are provided.

\section{BACKGROUND}

A review of literature to establish a point of departure for this research includes topics of Form Generation including Aesthetic Variables and Analytical Diagrams, Performance Evaluation run in a Multi-Objective Evolutionary Algorithm, Automatic Diagrams, and Form Diversity and Designers' Interaction. The following explains these concepts by reference to prior work.

\subsection{FORM GENERATION}

Parametric Modeling is a general methodology for defining models with constraints and variable parameters to enable generative form-making according to use of rules to automatically update design options based on changing parameters. ${ }^{2}$ Parametric design tools provide rapid iterations, often to achieve aesthetic form evaluation and to respond to environment-related requirements of energy savings, maximized daylight performance and respond to other criteria. This makes it significant to achieve performance-based design objectives by aiding in the generation of multiple, discrete solutions. ${ }^{9}$ The use of parametric modeling enables a designer to transition from designing one solution to designing a system that generates multiple solutions. ${ }^{30}$ It is important to note that shape grammar, a method developed to address formal computation for the algorithmic definition of languages of spatial designs, is parametric in specific ways. ${ }^{29}$

\subsubsection{FORMAL AESTHETIC VARIABLES IN FORM GENERATION}

Despite the view that aesthetics are idiosyncratic, general principles for building forms do exist. ${ }^{25}$ Primarily, two notions of aesthetics have been identified: (1) formal, concerned with structure of forms or formal aesthetics, (2) semantic or symbolic aesthetics, related to human responses to the form content, and the individual's internal representation of the building's meaning. ${ }^{25,22}$ Factors of formal aesthetics include shape, proportion, rhythm, scale, order, complexity, color, illumination, hierarchy, spatial relations, ambiguity, surprise, incongruity, and novelty. . $^{1722,25,33}$ This paper focuses on the language of formal aesthetics and does not address semantic aesthetics.

Various works have attempted to define styles/languages and their architectural aesthetics, such as The Classical Language Of Architecture ${ }^{31}$, The Modern Language of Architecture by Bruno Zevi ${ }^{39}$, The Language of Post-Modern Architecture by Charles Jencks ${ }^{18}$, and De-constructivist Architecture. ${ }^{20}$

Modernist aesthetics remain an important reference in contemporary 21st architectural aesthetics; recognized architects such as Frank Gehry, Sir Norman Foster, Peter Eisenman, Richard Meier and others utilize forms that owe largely to principles of modernism. ${ }^{11}$ The aesthetic references of the pioneers of modernism, Frank Lloyd Wright, LeCorbusier, and Ludwig Mies van der Rohe, are still influential in architectural education and practice. ${ }^{11}$ Bruno Zevi has elaborated seven "invariants" that characterize aspects of modernist design; the invariants help in key decisions regarding aesthetics without prescribing to particular styles. ${ }^{11,39}$ Our investigation utilizes Zevi's aesthetic variables of the language of modern architecture, described as follows: (1) functional listing, which is an activity in architectural design process in which the program and its desired performance is decomposed into units and sub-units, (2) asymmetry and dissonance is a dictate to avoid symmetry and follow program requirements and a dissonant pattern, (3) anti-perspective and three-dimensionality is the use of rich volumetric forms that are produced not for the sake of easiness to draw a perspective, but to satisfy functional and expressive objectives, (4) the syntax of fourdimensional decomposition is the quality of space that has indefinite boundaries to express unlimited expansion instead of enclosed space, (5) cantilever, shell, and membrane structure declares that structural innovation is a key element in modernism of which use of advanced structure engineering to create floating and breathtaking forms is pursued, (6) space in time emphasizes movement and change, and (7) reintegration of building, city, and landscape is the incorporation of inside-outside relationship in gradation and allows for multiple readings. ${ }^{11,39}$ 


\subsubsection{ANALYTICAL DIAGRAMS}

Analytical diagrams have been used to analyze architecture in various ways. In one interpretation, Palladio adds possibilities of superposition and overlapping of spatial layers into architectural space making up a "virtual" as opposed to literal condition. ${ }^{14}$ Another source for diagrams is the categories utilized by Kenneth Frampton in a comparative analysis to articulate principles of modern architecture. ${ }^{16} \mathrm{~A}$ computational approach to generating design forms that are similar to those created by famous architects, primarily at the conceptual or schematic level, posits that the vocabulary and rules for configuration may be captured. ${ }^{13}$

Analyzing a particular formal language can be considered as the activity of a structuralist who takes an object, decomposes it and finally recomposes it. ${ }^{5}$ The aim is to find intelligible dissection; dissection means separating each and every element of the analyzed object from each other to find reusable vocabularies. ${ }^{5}$ In this study, dissection is pursued through Zevi's variables in the form generation process.

\subsection{FITNESS FUNCTIONS AND PERFORMANCE EVALUATION}

Architectural design is characterized by involving many, often conflicting performance criteria. Recently, progress towards developing optimization methods has been investigated and integrated into architectural design research. ${ }^{1}$ Nevertheless, optimization research has been primarily directed to quantifiable building performance criteria such as construction costs, ${ }^{26}$ energy consumption and building systems, ${ }^{40}$ building envelopes, ${ }^{28}$ and daylight measures. ${ }^{32}$ It is recommended within the environmental performance evaluation platform to run daylight analysis and energy simulation simultaneously. ${ }^{27}$ This research considers energy and daylight as the performance criteria for optimization.

\subsubsection{MULTI-OBJECTIVE EVOLUTIONARY ALGORITHMS}

Real world design problems require simultaneous optimization of multiple, often conflicting, criteria or objectives. ${ }^{15}$ There are two main methods to solve Multi-Objective Optimization problems: (1) the weighted sum aggregation, in which the objectives are given weighting factors and the sum of weights is the composite objective function, (2) Pareto optimality, defined by engineer and economist Vilfredo Pareto, which is to find a family of promising non-dominated solutions known as the Pareto-front; each solution in this Pareto set is optimal when no improvement can be made in one objective without causing degradation in at least one of the remaining objectives. ${ }^{15}$

Evolutionary algorithms (EAs) are search algorithms that implement mating of pairs of solutions mimicking the natural selection process in evolution. ${ }^{21}$ An EA process begins with a population, often initiated randomly. ${ }^{21}$ Each candidate in the population is tested against fitness functions to determine whether it is an attractive candidate. The population is culled to include only attractive candidates, which are then combined to produce the next generation population. This new population is then used as the initial population in the EA process and the process repeats until a satisfactory solution is found. The operations of EAs exploit the characteristics of good solution according to different objectives and create new non-dominated solutions in parts of the Pareto front; such characteristics make EAs the most attractive approach to MOO problems. A Multi-Objective Evolutionary Algorithm (MOEA) has employed Pareto optimality to assist the optimization of all the objectives. ${ }^{15}$ In this work, we will use a MOEA-based tool for optimization.

\subsubsection{AUTOMATIC DIAGRAMS}

Automatic diagrams refer here to diagrams that are automatically generated for each form in the candidate set. The diagrams can be produced without human intervention based on the values chosen for architectural parameters. ${ }^{12}$ The change in parameters during the EA run causes change in the candidate form, and thus its diagram. The diagrams are automatically updated. Automatic diagrams are abstract representations of the candidates, useful for designers to evaluate aesthetics of the generated forms, filtering the forms according to their diagrams. If the diagrams are compliant with the stylistic preference, the candidate can be chosen by the designer, and inserted in a new run of EA. Analytical diagrams are helpful in the first phase of form generation, while automatic diagrams are important to choose the final solutions, post to optimization. Ultimately, the aim is to produce automatic diagrams to facilitate aesthetic evaluation, cull some solutions and introduce the reduced set into a new optimization run.

\subsection{FORM DIVERSITY AND DESIGNERS' INTERACTION}

To produce a disparate or diverse set of design solutions, optimization methods may be directed to eliminate overly similar solutions, ${ }^{8,24}$ and avoid halting on a local optima by scattering the search widely in the solution space. Importantly, a successful run of EAs requires a diverse set of design solutions to increase the possibility of finding a very high performance solution; a measure of diversity can be utilized to penalize similar options and quantify diversity of each set of solutions. ${ }^{8}$ One approach is to use a mixed mode method in which the user/designer can interact with optimization or let the EAs take on pure optimization in a back and forth process to choose the potential parents based on the designers' preferences. ${ }^{24}$ This interaction can reduce the fatigue associated with too many solutions, while maintaining highly varied candidates. ${ }^{24}$

\section{METHODS}

Our research method consists of the following processes: investigating, prototyping, and testing the prototype. 
Primarily, we aim to enhance optimization methods to include tasks to insert aesthetic variables into form making, increase diversity among candidates, and include choice of solutions based on aesthetic criteria, with the help of automatic diagrams. A prototype is being developed utilizing a test-case to exercise this prototype. Production of the prototype involves three main phases. (Figure 1). In Phase 1 (form generation), analytical diagrams of a test case work of architecture are created leading to a synthetic model that can be changed by changing parameters. Phase 2 (optimization) runs a generative system to evolve optimal solutions according to their performance feedback, and Phase 3 (form comparison) leads to highly distinguished forms. (Figure 1). Boxes in grey background are essential components that are the major contribution of this work to typical optimization environments.

This paper showcases the prototype framework, specifically focusing on form generation utilizing aesthetic variables and analytical diagrams of the test-case. The following is dedicated to the prototypical protocol and its phases.

\subsection{PHASE 1: INITIAL POPULATION}

For generating the form, design constraints must be defined such as: building site, program, design objectives, etc. As for utilizing aesthetic variables, we are working on two processes: analytical phase, and synthetic.

\subsubsection{ANALYTICAL PROCESS}

The analytical process in this experiment applies Zevi's aesthetic variables to a test case, the Atheneum Building of Richard Meier, located in New Harmony, Indiana (19751979), to identify its syntactical formal language. The reason for selecting the test case is due to its distinctive composition, being an example of modern architecture, and its status as one of the most important works of Meier. It is important to note that Meier's formal language can make the analytical process feasible because the architect employs a well-defined style- a consistent use of elements that can be traced from one building to another. ${ }^{3}$ However, our investigation aims ultimately to utilize the general formal principles of modern architecture; thus, the focus is on Zevi's variables and their application to multiple test cases including this one.

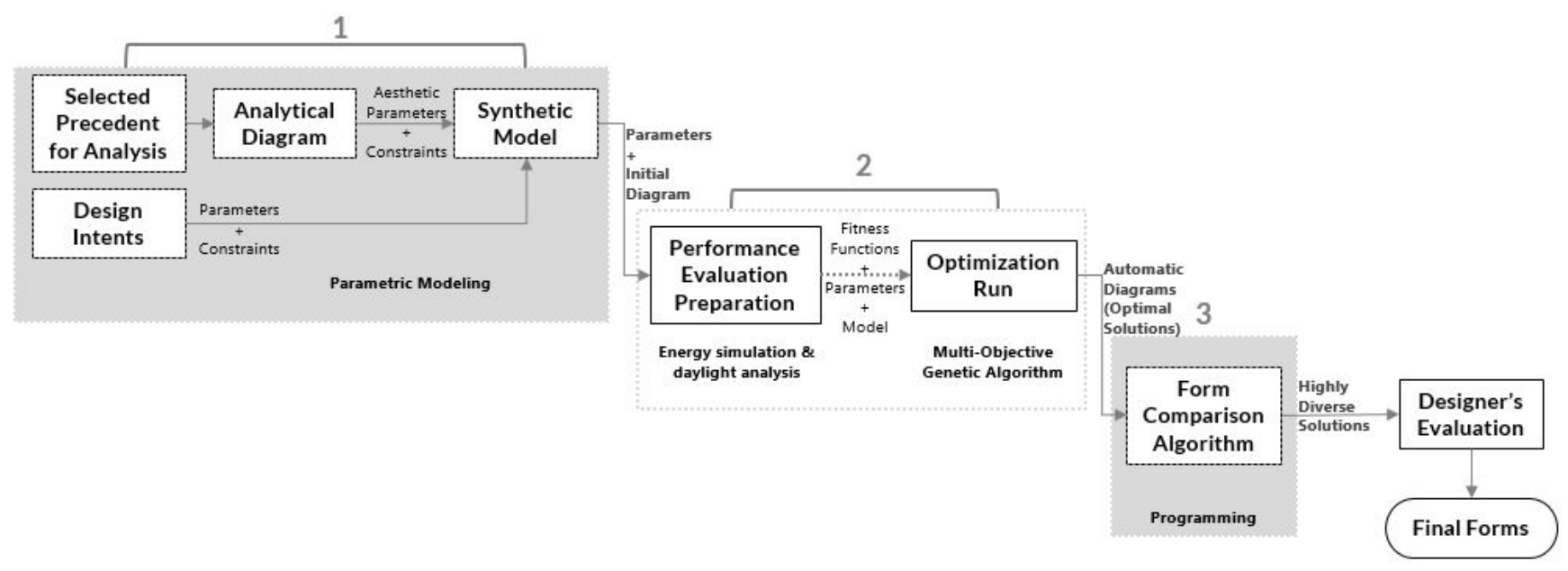

Figure 1: Research workflow illustrating phases and processes of prototype.

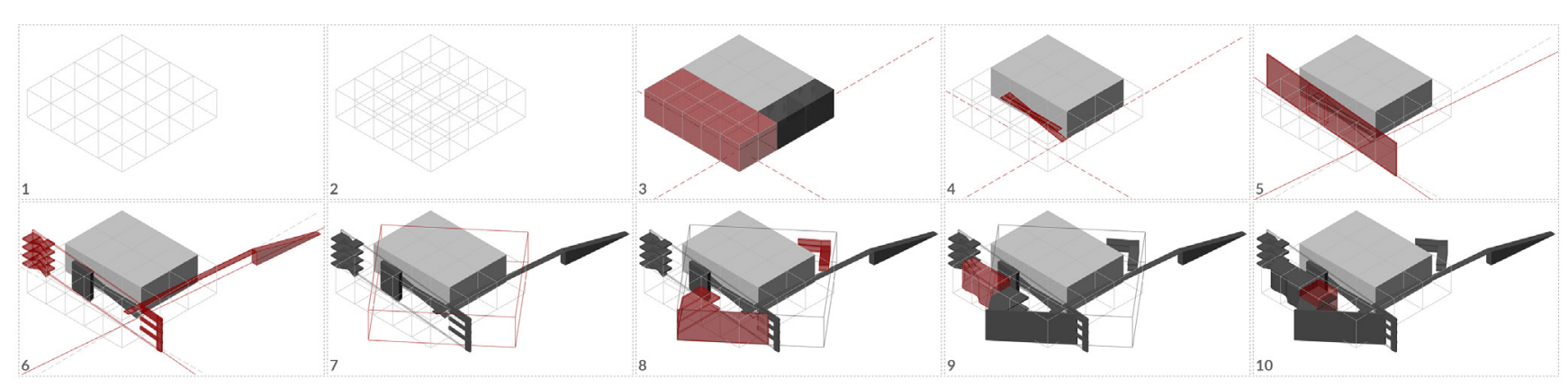

Figure 2: Steps of creating the Atheneum Building emphasizing the main syntactical characteristics and configuration. 
A computational model was created, representing the conceptual design of the Atheneum building, based on Geoffrey Baker's Design Strategies in Architecture, in which he analyzes the Atheneum. ${ }^{4,36}$ A program has been developed to model the Atheneum parametrically to understand the formal characteristics of the building. (Figure 2). First, an orthogonal grid was created with modular boxes (1), then the grid was modified to accommodate functionality (2), and program zones were attributed to the grid (3). Next, the main axis was emphasized by introducing a ramp attaching the auditorium (4). The axes were tilted ( 5 degrees), to orient the main routes ( $5 \& 6)$. A new rotated square that overlays the primary grid was introduced, stressed by a screen reorienting the entrance and stairs on the other side (8). A curved gallery was added, based on two units of the grid (9), and a light well was added (10). The Atheneum has more elements, and details, but in this analysis the focus was on the syntactical features and mass model configuration.

We identified four of Zevi's variables in this model. (Figure 3). Asymmetry and dissonance were apparent in the off centered axes that follow programmatic requirements (the auditorium is larger than the other spaces), and the tilted axes (1). The form employs antiperspective, evident through the use of spatially complex volumetric forms and the overlapping skewed grid (2). The concept of four dimensionality can be traced in the flow and indefinite boundaries of spaces, the openness and unlimited expansion (3). The variable of space in time appears in the distribution of circulation elements, the use of ramps and different moments of stairs to carefully direct the use of space in different times (4). (Figure 3).

\subsubsection{SYNTHETIC PROCESS}

In this phase, the model is parametrized to allow for changes that are governed by the aesthetic variables as constraints or/and parameters. The synthetic phase is still in progress, yet ideas have been elaborated on how to parametrize this case. For instance, the asymmetry concept should be used as a constraint to assure that there are no axes of symmetry. Antiperspective three-dimensionality can be programmed so that overlapping grid and complexity are maintained, through a range of parameters to control angles. Functional listing of spatial elements requires that the elements are clearly articulated one from another, while fourth dimensional decomposition requires defining elements to be eroded or dissolved to avoid a cellular composition.

\subsection{PHASE 2: FITNESS FUNCTIONS \& OPTIMIZATION}

In our prototype, design objectives of a minimum energy use and preferred daylight measure will be targeted as the performance objectives. In this phase, first, spacial descriptions are attributed to the model, adding programs (functions) to spaces, materials to all surfaces and assigning climatic conditions, all required for conducting energy simulation. In addition, assigning sky conditions, and adding sensors are often required for daylight grid-based analysis. Retrieving the daylight and energy analyses of the synthetic model will be connected to assigning the fitness functions. For the fitness functions, the minimal energy use, and a preferred daylight measure, such as illuminance values between (300-3000 lux) will be used as the fitness values. The search in the MOEA will be on finding the model options that are more fit in each optimization run, until Pareto optimization is met.

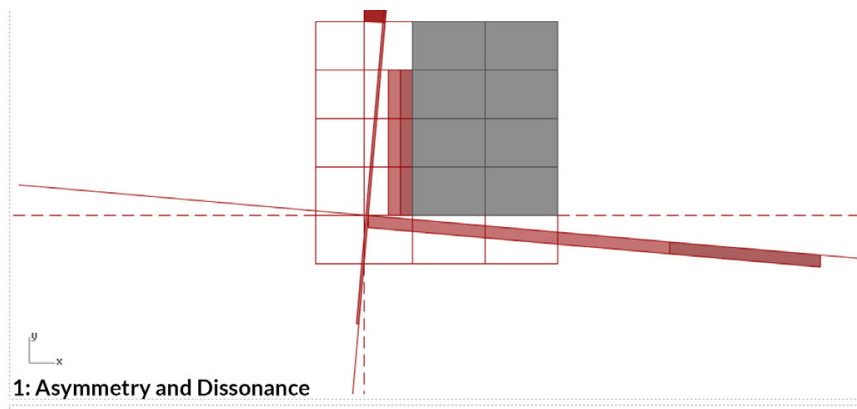

1: Asymmetry and Dissonance

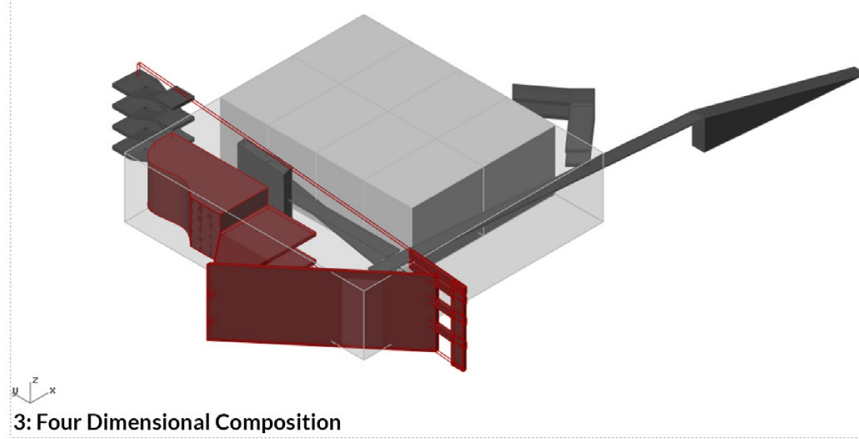

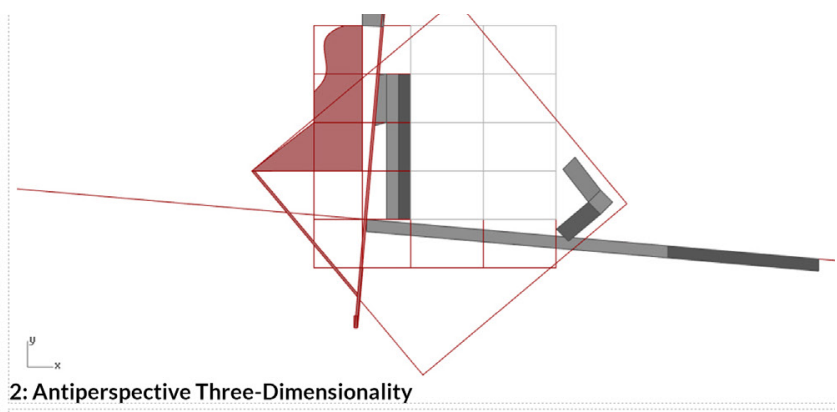

2: Antiperspective Three-Dimensionality

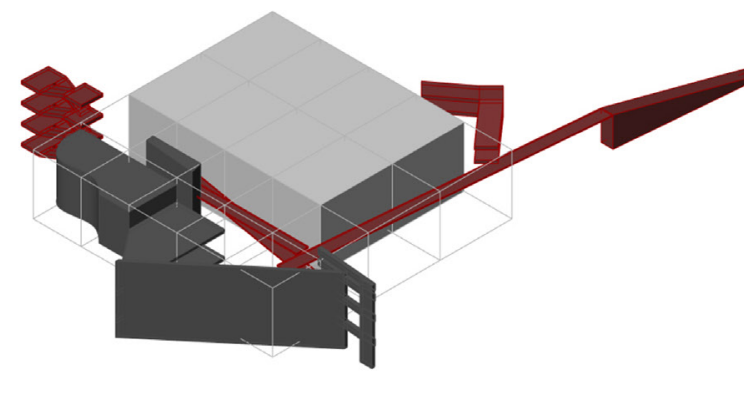

4: Circulation, Space in Time

Figure 3: Applying Bruno Zevi's variables to the test-case. 


\subsubsection{AUTOMATIC DIAGRAMS AND THE SOLUTION SET}

While the synthetic model represents the overall configuration and the main rules used, the resulting multiple possible alternatives generated from changing the parameters through the MOEA run are the automatic diagrams (optimal solutions). The purpose of using automatic diagrams is to aid in assessing the resulting forms and examine the diagrammatic qualities. This can facilitate the aesthetic evaluation of final solutions. The role of the automatic diagrams is to assist the designer, by inspecting them to eliminate some candidates either prior to running another iteration of the EA or in deciding not to run another iteration.

\subsection{PHASE 3: FILTERING THE SOLUTION SET}

In a prior work, post to optimization, a form comparison algorithm (software program) was developed and implemented into the system, applied to a grid-based pattern that was capable of generating a variety of alternatives. The algorithm calculates the form difference values between each pair of 100 optimal and near-optimal design solutions at generation 10. A threshold was set as a measure for form diversity, and out of the 100 solutions the algorithm selected 20 of highly diverse forms. ${ }^{38}$

This form comparison method will be implemented in the post-optimization phase in this work to articulate final solutions and accommodate designers' interaction and evaluation of forms in the system. After filtering the highly diverse candidates, the designer utilizes the automatic diagrams to assess those candidates.

\section{DISCUSSION \& CONCLUSIONS}

This study presents a shift from optimization design methods that deal with technical performance design objectives into optimization that incorporates objectives in aesthetics. A new architectural design optimization framework has been suggested and explained. The work is still in progress, and upon completion we plan to make the prototype available as an open-source framework usable in widely-available modeling programs.

For the next step, experiments that utilize additional recognized modern buildings as test cases for analysis are needed. A potential improvement is to test incorporating a fitness function for aesthetics. The simulation and optimization experiment in a previous work proved successful in evolving optimal solutions, with expected behavior of satisfying the energy and daylighting measures. The developed algorithm for form comparison has led to highly different forms, eliminating similar geometric forms. Nevertheless, further development is needed for the form comparison. Finally, after completing a fully working prototype, validation experiments are needed to test whether it is perceived useful in practical design problems.

\section{RESEARCH SIGNIFICANCE}

The significance of this work is to improve the capability of design methods in general and architectural design optimization in particular to integrate qualitative and quantitative evaluation of multiple design options. This research could lead to a new system for architectural design optimization by:

- Incorporating formal aesthetics into computer-aided building design platforms to fulfill the significant aesthetic criterion, often ignored in simulation and optimization platforms.

- Providing a prototype and platform that makes it feasible for designers to consider re-thinking current optimization models.

\section{ENDNOTES}

1 Aish, Robert, Al Fisher, Sam Joyce, and Andrew Marsh. "Progress Towards Multi-Criteria Design Optimisation Using Designscript with Smart Form, Robot Structural Analysis and Ecotect Building Performance Analysis." Paper presented at the Proceedings of ACADIA, 2012

2 Aish, Robert, and Robert Woodbury. "Multi-Level Interaction in Parametric Design." Paper presented at the Smart Graphics, 2005.

3 Al Assaf, Nancy, and Mark Clayton. "Representing the Aesthetics of Richard Meier's Houses Using Building Information Modeling." In ACADIA 17: Disciplines and Disruption, Proceedings of the 37th Annual Conference of the Association for Computer Aided Design in Architecture (ACADIA). MIT, Cambridge, MA, 2017.

4 Baker, Geoffrey H. Design Strategies in Architecture: An Approach to the Analysis of Form. Taylor \& Francis, 2003.

5 Barthes, Roland. "The Structuralist Activity." In Critical Essays, 213-20. (Evanston, Illinois: Northwestern Univerity Press, 1972).

6 Booth, Geoffrey, M Clayton, and JB Kim. “A Framework for Designing Sustainable Real Estate Developments Using Quadruple Net Value Analysis and Building Information Modelling." Paper presented at the CIB World Congress, Brisbane, Australia, 2013.

7 Brown, Nathan C, and Caitlin T Mueller. "Design for Structural and Energy Performance of Long Span Buildings Using Geometric Multi-Objective Optimization." Energy and Buildings 127 (2016): 748-61.

8 Brown, Nathan, Stavros Tseranidis, and Caitlin Mueller. "Multi-Objective Optimization for Diversity and Performance in Conceptual Structural Design." Proceedings of the International Association for Shell and Spatial Structures (IASS) (2015).

9 Caplan, Bill. "Parametric Design's Greatest Value to Architecture Is to Attain Eco-Sustainability." The Architectural Review, EMAP Architecture (2011).

10 Chouchoulas, Orestes. "Shape Evolution: An Algorithmic Method for Conceptual Architectural Design Combining Shape Grammars and Genetic Algorithms." University of Bath, 2003.

11 Clayton, Mark. "Diagramming Aesthetics: Modernism and Architecture in the 21st Century." In Theory and Application of Diagrams: First International Conference, Diagrams 2000 Edinburgh, Scotland, Uk, September 1-3, 2000 Proceedings, edited by Michael Anderson, Peter Cheng and Volker Haarslev, 257-70. Berlin, Heidelberg: Springer Berlin Heidelberg, 2000.

12 Clayton, Mark. "Standard Visualizations of Architectural Concepts in Support of Architectural Education." Paper presented at the Proceedings of the 2013 ARCC Spring Research Conference, Architectural Research Centers Consortium, The Visibility of Research, Charlotte, North Carolina, March 27 - 30, 2013.

13 Clayton, Mark, Ozan Ozener, Ehsan Barekati, and James Haliburton. "Signature Architecture Franchising: Improving Average Architecture Using Bim." Paper presented at the Proceedings of Digital Aptitudes and other openings, ACSA 100th Annual Meeting, Boston, MA, March 1-4, 2012

14 Eisenman, Peter, and Matt Roman. Palladio Virtuel. Yale University Press, 2015.

15 Fonseca, Carlos M, and Peter J Fleming. "Genetic Algorithms for Multiobjective Optimization: Formulationdiscussion and Generalization." Paper presented at the Icga, 1993.

16 Frampton, Kenneth, and Ashley Simone. A Genealogy of Modern Architecture: Comparative Critical Analysis of Built Form. Edited by Ashley Simone. (Zürich: Lars Müller Publishers, 2015).

17 Groat, Linda N, and Carole Després. "The Significance of Architectural Theory 
for Environmental Design Research." In Advances in Environment, Behavior, and Design, 3-52: Springer, 1991.

18 Jencks, Charles. The Language of Post-Modern Architecture. 6th Ed. Charles Jencks. (New York: Rizzoli, 1991).

19 Jerke, Dennis, Douglas R. Porter, and Terry J. Lassar. Urban Design and the Bottom Line: Optimizing the Return on Perception. Dennis Jerke, Douglas R. Porter, Terry J. Lassar. Washington, D.C.: ULI-Urban Land Institute, [2008] 2008. Bibliographies.

20 Johnson, Philip, and Mark Wigley. Deconstructivist Architecture. Little Brown and Company, 1988.

21 Konak, Abdullah, David W Coit, and Alice E Smith. "Multi-Objective Optimization Using Genetic Algorithms: A Tutorial." Reliability Engineering \& System Safety 91, no. 9 (2006): 992-1007.

22 Lang, Jon. "Symbolic Aesthetics in Architecture: Toward a Research Agenda." Environmental Aesthetics: Theory, Research, and Application (1988): 45-55.

23 Laseau, Paul. Graphic Thinking for Architects and Designers. John Wiley \& Sons, 2001.

24 Marsault, Xavier. "A Multiobjective and Interactive Genetic Algorithm to Optimize the Building Form in Early Design Stages." Paper presented at the 13th Conference of International Building Performance Simulation Association 2013

25 Nasar, Jack L. "Urban Design Aesthetics: The Evaluative Qualities of Building Exteriors." Environment and Behavior 26, no. 3 (May 1, 1994 1994): 377-401.

26 Radford, Anthony D, and John S Gero. Design by Optimization in Architecture, Building, and Construction. John Wiley \& Sons, Inc., 1987.

27 Roudsari, Mostapha Sadeghipour, Michelle Pak, and Adrian Smith. "Ladybug: A Parametric Environmental Plugin for Grasshopper to Help Designers Create an Environmentally-Conscious Design." Paper presented at the Proceedings of the 13th International IBPSA Conference Held in Lyon, France Aug, 2013.

28 Shea, Kristina, Andrew Sedgwick, and Giulio Antonuntto. "Multicriteria Optimization of Paneled Building Envelopes Using Ant Colony Optimization." In Intelligent Computing in Engineering and Architecture, 627-36: Springer, 2006.

29 Stiny, George. "Introduction to Shape and Shape Grammars." Environment and Planning B: Planning and Design 7, no. 3 (1980): 343-51.

30 Stocking, Angus W. "Generative Design Is Changing the Face of Architecture'." Building Design (2009).

31 Summerson, John. The Classical Language of Architecture. MIT Press, 1963.

32 Torres, S, and Yuzo Sakamoto. "Facade Design Optimization for Daylight with a Simple Genetic Algorithm." Paper presented at the Proceedings of Building Simulation, 2007

33 Wohlwill, Joachim F. "Environmental Aesthetics: The Environment as a Source of Affect." Human Behavior and Environment: Advances in Theory and Research 1 (1976): 37-86

34 Wurzer, G, U Pont, WE Lorenz, and A Mahdavi. "Coupling Building Morphology Optimization and Energy Efficiency-a Proof of Concept." Paper presented at the CESBP Central European Symposium on Building Physics AND BauSIM 2016, Dresden, Germany, 2016.

35 Yan, W, M Rahmani Asl, Z Su, and J Altabtabai. "Towards Multi-Objective Optimization for Sustainable Buildings with Both Quantifiable and NonQuantifiable Design Objectives." In Sustainable Human-Building Ecosystems, 223-30, 2015

36 Yan, Wei. "Design Thinking Visualization." In CAADRIA 2007 [Proceedings of the 12th International Conference on Computer Aided Architectural Design Research in Asia], 219-26. Nanjing, China, 2007.

37 Yang, Rui, and Lingfeng Wang. "Multi-Objective Optimization for DecisionMaking of Energy and Comfort Management in Building Automation and Control." Sustainable Cities and Society 2, no. 1 (2012): 1-7.

38 Yousif, Shermeen, Wei Yan, and Charles Culp. "Incorporating Form Diversity into Architectural Design Optimization." Paper presented at the ACADIA 17: Disciplines and Disruption, Proceedings of the 37th Annual Conference of the Association for Computer Aided Design in Architecture (ACADIA), 2017.

39 Zevi, Bruno. The Modern Language of Architecture. Seattle: University of Washington Press, 1978.

40 Zhang, Yinping, Kunping Lin, Qunli Zhang, and Hongfa Di. "Ideal Thermophysical Properties for Free-Cooling (or Heating) Buildings with Constant Thermal Physical Property Material." Energy and Buildings 38, no. 10 (2006): 1164-70. 